\title{
Caring for the Elderly Parents: The Role of Daughters
}

\author{
Songze $\mathrm{Chu}^{1, *}$ \\ ${ }^{1}$ Experimental High School Attached to Beijing Normal University \\ ${ }^{*}$ Corresponding author. Email: jasonchusz@outlook.com
}

\begin{abstract}
With the development of a modern social economy and culture in China, women's role in elderly care has become more important. However, limited amount of research has been completed on how exactly women are contributing to elderly care in a family. In order to further the knowledge of elderly care and to better understand Chinese daughters' role in providing elderly care to parents, this study utilized a cross-sectional survey methodology with a sample of 302 to examine how adult daughters support their elderly parents. The questionnaire data was input into SPSS and analyzed with t-tests and correlation analysis. The result shows that: (1) Compared to sons, daughters provide more emotional support in terms of listening to parents' thoughts and talking to parents about life. (2) Daughter's age is positively correlated with the support they give to parents. (3) Daughter's distance from parents is negatively correlated with the support they give to parents.
\end{abstract}

Keywords: Elderly Care, Daughters' Role, Gender Difference, Family Support.

\section{INTRODUCTION}

According to Chinese traditional culture, "Married daughters are like spilled water and do not belong to her parents' family anymore" [1]. Therefore, the responsibility of supporting parents was mainly borne by the son rather than the daughter. This tradition had continued for thousands of years in Chinese culture. However, the traditional mode of supporting the parents only by sons has begun to change significantly.

The role of women in caring for the elderly has evolved over thousands of years and seen significant changes in recent societies. Traditionally, the dominant position of the father in the family power structure was enough to support the old-elderly parents. In addition, family succession was particularly preferred to sons. At the old age, the son not only inherited the family property, but also had to fulfill the elderly-care obligation; the daughter did not have the right to inherit the family property, and the parents did not expect the daughter to support them [2]. Generally, the daughter shall take good care of her parents in law and taking care of her parents was based on gratitude [3]. The distribution of responsibility of providing against old age was mainly among the sons. Women lacked enough voice in the distribution of responsibility of providing against old age, and generally followed the arrangement of their husbands [2]. With the family transformation and social change, the pressure to support elderly parents by sons has been increasing, which gradually activates the family politics in the allocation of responsibility of supporting elderly parents and tends to expand the responsibility for supporting parents. In this case, daughters begin to participate in supporting her parents for backup [3]. As time passed women's economic and family status have changed dramatically, their role changed from being regarded as "a person with a different surname" to being "also a descendant". A daughter's support for her parents was mainly out of love and conscience in the past. But now daughters have the duty to support their parents, and they have begun to play a core role in supporting their elderly parents. Daughter's support for parents has gradually become accepted by the society, and the role of daughters in supporting parents' life has been paid more and more attention. There are three main factors that accelerated providing elderly-care by daughters, namely policy change, culture change, and change in gender roles.

\subsection{Policy Change}

With the implementation of the family planning policy, the family structure and costs of supporting individuals in old age has changed greatly; sons face more and more pressure to support elderly parents [4]. In this context, the daughters' participation complements 
the resources of family elderly care support and may reduce the pressure of sons. In addition, due to the onechild policy (From 1982, the birth of new child is limited by the Chinese government. From 1982 to 2016, each family was allowed to have only one child. From 2016 to 2021, every family can have two children. After 2021, every family can have three children.), the only-child plays an inevitable role in supporting their parents. It has become a legal obligation for daughters to provide support for their elderly parents [5].

\subsection{Culture Change}

Culture change also has a great impact on the provision of elderly-care by women. Now many parents' dependence and expectation on their daughters has grown, and their daughters have the additional role expectation of filial piety (Take care of, respect and obey parents) to their parents [6].

\subsection{Change in Gender Roles}

With the improvement of women's social and economic status, women have gradually taken on the dominant role in the family power structure. Change of social environment is another important reason that puts women in main role of elderly-care [3]. With the increase in women's social status, they have obtained equal opportunities for educational and vocational skills training, leading to more employment and development opportunities. As a result, women are experiencing increased economic independence enabling them to provide parents economic supports [3]. All these changes in the position of men and women established the economic and social foundation for daughters to support their elderly parents.

With the development of the modern social economy and culture, the population aging trend has intensified the various living demands of the elderly. The family structure has become smaller and more concentrated and women's role in elderly care has become more important. However, there is only a limited amount of research that has been completed on how women are contributing to elderly care in a family. That is, are women providing more economic or emotional support to their elderly parents than sons? The aim of this exploratory research study is to further the knowledge of elderly care and to better understand Chinese daughters' roles in providing elderly care to their parents.

\section{METHOD}

This study utilized a cross-sectional survey methodology with a sample of 302 adults with at least one living parent to examine how adult daughters support their elderly parents. We tested six primary hypotheses:

(H1) The higher the daughters' educational level, the more likely they will be to support their elderly parents

(H2) The closer the daughters live to their elderly parents, the more likely they will be to support their elderly parents

(H3) The older the daughters are, the more likely they will be to support their elderly parents

(H4) The higher the daughters' income, the more likely they will be to economically support their elderly parents

(H5) Women residing in urban areas are generally more supportive of their parents than women living in rural regions.

(H6) Compared to sons, daughters provide more emotional support and less financial support to elderly parents

Participants were obtained through the using of nonprobability sampling methods. Participants were recruited through WeChat over the course of three days with convenience sampling. Quantitative data was collected through the distribution of anonymous self-administered survey questionnaires through WeChat survey platform. The investigator-developed survey included demographic information and questions assessing adult children's support to their elderly parents. Demographic information included gender, age, level of education, occupation, income level, family harmony and relationship with parents. Questions assessing adult children's support to their elderly parents included three major aspects, namely financial support, household assistance, and emotional support between generations. Financial support is measured by the extent to which participants report paying for parents' living expenses or medical bills; household assistance is measured by helping parents with housework or chores, while emotional support is measured by listening to parents' thoughts or talking to parents about respondents' life. By looking into these aspects, a more comprehensive picture of elderly care support by daughters in China can be gained.

In the study, support to older parents was operationalized into six categories: (1) Paying for parents' living expenses; (2) Paying for parents' medical bills; (3) Helping parents with housework; (4) Helping parents with chores; (5) Listening to parents' thoughts face to face or via phone calls; and (6) Talking to parents about respondents' life face to face or via phone calls. The first two questions reflect financial support to elderly parents, the following two questions reflect household assistance to elderly parents and the last two questions reflect emotional support to elderly parents. Each of the six domains of the elderly care practice was asked by "In the past year, how often have you provided the following support to your own parents?" A five-point scale was used with one representing "never" and five "very often".

Since the topic of the study was elderly care support 
provided to aging parents, only subjects with at least one living aging parent were included. After excluding samples that do not match the criteria $(n=5)$, the final sample for the study was 302 respondents, including 214 females and 88 males. The survey was administered using WJX, an online survey software system, and required an average of 10 minutes to complete. Prior to participation, consent was obtained through an online description of the study and the option to participate or withdraw from the study.

\section{DATA ANALYSIS}

Upon completion of the study, the questionnaire data was imported into SPSS and analyzed by t-tests and correlation analysis. One focus of this research is to determine whether there is a difference in the way daughters and sons support their older parents, so six independent t-tests are performed to examine gender difference on providing elderly care. Another focus of this research is to determine whether there is a difference in the way daughters from urban and rural area support their older parents, so six t-tests are performed to see the difference between urban and rural areas on daughters' providing elderly care. Correlation analysis is conducted to detect whether daughters' demographics (educational level, distance from parents, age and annual income) have a relationship with the elderly care they provide to their parents.

\section{RESULTS}

\subsection{Correlation between daughter's demographics and support to parents}

The results of the correlation analysis between daughter's demographics (educational level, distance from parents, age and annual income) and support to parents were summarized in Table 1.

Table 1. Pearson Correlation Between Daughter's Demographics and Support to Parents

\begin{tabular}{|c|c|c|c|c|c|c|c|}
\hline & & $\begin{array}{l}\text { Paying for } \\
\text { living } \\
\text { expenses }\end{array}$ & $\begin{array}{c}\text { Paying for } \\
\text { medical bills }\end{array}$ & $\begin{array}{c}\text { Helping } \\
\text { with } \\
\text { housework }\end{array}$ & $\begin{array}{c}\text { Helping with } \\
\text { chores }\end{array}$ & $\begin{array}{l}\text { Listening to } \\
\text { parents' } \\
\text { thoughts }\end{array}$ & $\begin{array}{c}\text { Talking to } \\
\text { parents about } \\
\text { own life }\end{array}$ \\
\hline \multirow{2}{*}{$\begin{array}{c}\text { Educational } \\
\text { level }\end{array}$} & Coefficient & -0.006 & -0.060 & -0.054 & -0.015 & 0.089 & 0.102 \\
\hline & $p$ value & 0.935 & 0.379 & 0.431 & 0.824 & 0.194 & 0.136 \\
\hline \multirow{2}{*}{$\begin{array}{c}\text { Distance } \\
\text { from parents }\end{array}$} & Coefficient & -0.066 & -0.073 & $-0.491^{\star * *}$ & $-0.492^{\star \star \star}$ & $-0.229^{\star \star *}$ & -0.106 \\
\hline & $p$ value & 0.335 & 0.285 & 0.000 & 0.000 & 0.001 & 0.122 \\
\hline \multirow{2}{*}{ Age } & Coefficient & 0.054 & $0.185^{\star \star}$ & $0.246^{\star \star}$ & $0.219^{\star \star}$ & $0.205^{\star \star}$ & 0.043 \\
\hline & $p$ value & 0.432 & 0.007 & 0.000 & 0.001 & 0.003 & 0.531 \\
\hline \multirow{2}{*}{$\begin{array}{l}\text { Annual } \\
\text { income }\end{array}$} & Coefficient & $0.224^{\star \star}$ & $0.232^{\star \star}$ & -0.006 & 0.072 & 0.037 & 0.012 \\
\hline & $p$ value & 0.001 & 0.001 & 0.928 & 0.297 & 0.594 & 0.857 \\
\hline
\end{tabular}

Educational level: Six categories were provided ranging from: 1 = primary school; 2 = middle school (or technical middle school); $3=$ high school; $4=$ junior college; 5 = bachelor's degree; and $6=$ advanced degree. According to the Pearson correlation results, coefficients for six aspects of support given to parents were all close to zero. No significant correlation coefficient was found between educational level and support to elderly parents.

Distance from parents: Seven categories were provided ranging from: 1 = living together; 2 = walking distance; $3=0.5$ hour by car; $4=0.5$ to 1 hour by car; $5=$ 1 to 2 hours by car; $6=$ more than 2 hours by car; and 7 $=$ in different cities. Significant negative correlation coefficient was found between distance from parents and helping with housework, helping with chores, and listening to parents' thoughts, meaning that the farther away a child moved from their parent, the less likely they were to help their parent with housework, etc.

Age: Respondents' age ranged from: $1=$ under 20 years; $2=20$ to 29 years; $3=30$ to 39 years; $4=40$ to 49 years; $5=50$ to 59 years; and $6=60$ years and above. Significant positive correlation coefficient was found between age and paying for medical bills, helping with housework, helping with chores, and listening to parents' thoughts, meaning that the older children were, the more likely they were to report paying for their parents' medical bills, etc. 
Annual income: Income was measured according to individual annual income. The scores were divided into six groups: $1=$ no income; $2=$ less than 100,000 Yuan; 3 $=100,000$ to 300,000 Yuan; $4=300,000$ to 500,000 Yuan; $5=500,000$ to $1,000,000$ Yuan; and $6=$ more than $1,000,000$ Yuan. Significant positive correlation coefficient was found between annual income and paying for living expenses, and paying for medical bills, meaning that the more income a child made, the more likely they were to report helping their parent with expenses.

\subsection{Difference between urban and rural areas on daughters' providing elderly care}

The results of six t-tests comparing the difference between urban and rural areas on daughters' providing elderly care is summarized in Table 2 .

Table 2. Urban/Rural Difference on Daughters' Providing Elderly Care

\begin{tabular}{|c|c|c|c|c|c|c|}
\hline & \multicolumn{4}{|c|}{ Area } & \multirow{3}{*}{$t$} & \multirow{3}{*}{$p$} \\
\hline & \multicolumn{2}{|c|}{$\operatorname{Urban}(n=201)$} & \multicolumn{2}{|c|}{ Rural $(n=13)$} & & \\
\hline & Mean & SD & Mean & SD & & \\
\hline $\begin{array}{l}\text { Paying for parents' living } \\
\text { expenses }\end{array}$ & 2.75 & 1.49 & 3.38 & 1.26 & -1.506 & 0.134 \\
\hline Paying for parents' medical bills & 2.34 & 1.53 & 2.85 & 1.63 & -1.157 & 0.249 \\
\hline Helping parents with housework & 2.22 & 1.31 & 2.08 & 0.86 & 0.573 & 0.575 \\
\hline Helping parents with chores & 2.84 & 1.29 & 2.69 & 1.03 & 0.406 & 0.685 \\
\hline $\begin{array}{l}\text { Listening to parents' thoughts } \\
\text { face to face or via phone calls }\end{array}$ & 3.56 & 1.07 & 3.23 & 1.17 & 1.063 & 0.289 \\
\hline $\begin{array}{l}\text { Talking to parents about } \\
\text { respondents' life face to face or } \\
\text { via phone calls }\end{array}$ & 3.57 & 1.08 & 3.62 & 1.04 & -0.140 & 0.889 \\
\hline
\end{tabular}

Participants were asked if they were from the urban or rural area from China. Two hundred and one female respondents were from urban area while thirteen female respondents were from rural area. Through the analysis, the study compared whether differences existed between urban and rural areas in terms of daughters caring for the elderly. For questions about "paying for parents' living expenses", "paying for parents' medical bills" and "talking to parents about respondents' life face to face or via phone calls", the mean values for female respondents from rural areas are higher. For the other three questions, the mean was higher for urban female respondents.
However, the t-tests revealed that for all six questions, there was no statistically significant difference among female respondents from urban and rural areas in terms of the support they providing to their parents. This may be due to the small sample size of rural participants $(n=13)$.

\subsection{Gender difference on support to elderly parents}

The results of 6 t-tests about gender difference on providing elderly care were summarized in Table 3. 
Table 3. Gender Difference in Supporting Elderly Parents

\begin{tabular}{|c|c|c|c|c|c|c|}
\hline & \multicolumn{4}{|c|}{ Gender } & \multirow{3}{*}{$t$} & \multirow{3}{*}{$p$} \\
\hline & \multicolumn{2}{|c|}{$\operatorname{Male}(n=88)$} & \multicolumn{2}{|c|}{ Female $(n=214)$} & & \\
\hline & Mean & SD & Mean & SD & & \\
\hline $\begin{array}{c}\text { Paying for parents' living } \\
\text { expenses }\end{array}$ & 2.50 & 1.44 & 2.79 & 1.49 & -1.529 & 0.127 \\
\hline Paying for parents' medical bills & 2.30 & 1.58 & 2.37 & 1.53 & -0.376 & 0.707 \\
\hline Helping parents with housework & 2.02 & 1.20 & 2.21 & 1.29 & -1.202 & 0.230 \\
\hline Helping parents with chores & 2.36 & 1.15 & 2.83 & 1.27 & -2.984 & $\begin{array}{c}0.003^{*} \\
*\end{array}$ \\
\hline $\begin{array}{l}\text { Listening to parents' thoughts } \\
\text { face to face or via phone calls }\end{array}$ & 3.16 & 1.10 & 3.54 & 1.07 & -2.762 & $\begin{array}{l}0.006^{*} \\
*\end{array}$ \\
\hline $\begin{array}{l}\text { Talking to parents about } \\
\text { respondents' life face to face or via } \\
\text { phone calls }\end{array}$ & 3.03 & 1.17 & 3.57 & 1.08 & -3.870 & $\begin{array}{l}0.000^{*} \\
*\end{array}$ \\
\hline
\end{tabular}

\subsection{Gender difference on financial support to elderly parents}

Two questions about "paying for parents' living expenses" and "paying for parents' living expenses" were aimed at determining respondents' financial supports to their elderly parents. The two t-tests were aimed at determining if there was a gender difference on providing financial support to elderly parents. Although mean values of the two questions for female are both higher than those of the male respondents, the t-tests revealed that the results are not statistically significant. There are no gender differences in financial support to elderly parents.

\subsection{Gender difference on household assistance to elderly parents}

Two questions about "helping parents with housework" and "helping parents with chores" were aimed at determining respondents' frequency of providing household assistance to their elderly parents. The two t-tests were aimed at determining if there was a gender difference on household assistance to elderly parents. For the "helping parents with housework" question, although the mean value for female respondents is higher than that of the male respondents, the t-test revealed that there are no gender differences. For the "helping parents with chores" question, the t-test resulted in a statistically significant difference, $\mathrm{t}=-2.984, \mathrm{p}=$ 0.003. Compared to sons, daughters reported that they more frequently help parents with chores.

\subsection{Gender difference on emotional support to elderly parents}

This study also looked at adult children's emotional support to elderly parents. Participants were asked to answer two questions related to emotional support: "listening to parents' thoughts face to face or via phone calls" and "talking to parents about respondents' life face to face or via phone calls". For the first question the t-test determined that there was a statistically significant difference between gender in frequency of listening to parents' thoughts face to face or via phone calls, $t=$ $2.762, p=0.006$. It was determined that daughters were more likely to listen to parents' thoughts face to face or via phone calls. For the second question the t-test also determined that gender is significant when it comes to talking to parents about respondents' life face to face or via phone calls. For this question $\mathrm{t}=-3.870, \mathrm{p}=0.000$. So, in general daughters provide more emotional support to elderly parents compared with sons.

\section{CONCLUSION}

In this study, we compared the differences between daughters and sons in terms of the financial support, household assistance and emotional support they provide to their parents. We analyzed the differences between daughters in urban area and rural area in terms of the support they provide. We also analyzed the relationship between daughters' demographics (educational level, distance from parents, age and annual income) and the support they provide. Six hypotheses were tested.

Hypothesis one, that the higher the daughters' educational level, the more support they provide to their elderly parents, is not supported. There is no significant correlation between daughter's educational level and 
support to parents. Being more educated doesn't necessarily mean that they will support their parents more. However, this is contradicted to the current literature. "Children's education level has a negative impact on daily care and spiritual comfort, and a positive impact on economic support. The higher the children's education level, the lower the level of daily care and spiritual comfort, the higher the level of economic support [7]."

Hypothesis two, that the closer the daughters live, the more support they provide to their elderly parents, is partially supported. There is negative correlation between daughter's distance from parents and the household assistance they provide to parents, indicating that the greater the distance, the less children help their parents with housework. This makes sense since the further children are from their parents, the more difficult it is for them to help parents with housework and chores.

Hypothesis three, the older the daughters are, the more support they provide to their elderly parents, is partially supported. Daughter's age is positively correlated with the support they give to parents in four out of the six aspects (paying for medical bills, helping with housework, helping with chores, listening to parents' thoughts). Indicating that the older a child is, the more likely he or she is to help parents pay for medical bills, helping with housework, helping with chores, listening to parents' thoughts. This might be caused by the fact that the older daughters get, the older their parents are. Parents will need more help from their daughters.

Hypothesis four, which suggests that the higher the daughters' income, the more economically support they provide to their elderly parents, is also partially proved. Daughters' income is positively correlated with the financial support they provide to parents, meaning that as daughters income increases, the likelihood that they will financially support their parents increases. Although, traditionally, women in China held little responsibility in caring for their elderly parents because of the traditional Chinese belief of filial piety, our study suggests that with modern ideology and improved resources, women's supportive behavior toward parents has changed, and it may become increasingly common for daughters to perform filial acts, just as their male counterparts do. In some of the aspects, such as providing emotional support, women are taking on a more prominent role than men. The study also revealed that different demographic factors are related to the support daughters provide to their parents. Age and income have positive correlation with elderly care support while distance from parents has negative correlation with elderly care support.

Hypothesis five, which is, urban women are generally more supportive of their parents than rural women, is not supported. Difference between urban and rural areas on daughters' providing elderly care is not statistically significant. From the sample mean, rural daughters even provide more frequent financial support to their parents. While this result was not statistically significant in our sample, future studies should continue to understand this question. Our rural sample size was small $(n=13)$, which might have limited our statistical power and ability to fully understand this question.

Hypothesis six, that is, compared to sons, daughters provide more emotional support and less financial support to elderly parents, is partially supported. We found that compared to sons, daughters provide more emotional support in terms of listening to parents' thoughts and talking to parents about own life. This could be related to the fact that women are more likely to communicate with their parents and retain contact. However, gender differences in the provision of financial assistance are statistically not significant. There might be two reasons that caused this. First, most participants for this study were from big cities. Since the earning gap between men and women in the labor market in big cities are small, the financial assistance they provide to parents may also be similar. This is supported by the current literature. "The bigger the city, the smaller the gender wage gap [8]." Second, it was the frequency of financial support the respondents gave that we compared. Although the frequency difference is small, the amount of money sons and daughters provide may still be different.

A few limitations have been raised that need to be addressed in future work. First, for the convenience of this research, the questionnaires were sent to sons and daughters directly but not to their parents (People in old age may face difficulty to fill out our questionnaire online). More data from the parents' perspective should be collected to conduct more in-depth research on the topic. Another limitation is that support to parents contains so many factors that are unquantifiable, and the information reported by the respondents is largely subjective (a five-point scale was used in the study to reflect the support children give), so the results should be interpreted with a degree of caution. If we want to use a regression model to determine the influence of different factors on daughter's support given to parents, more quantifiable statistics should be collected in the future. The distinction between housework and chores is a subjective understanding, and more restrictions and examples should be imposed in the investigation and research to help respondents define their differences and obtain more effective data. Finally, from the results of this study we see no difference between rural and urban areas in China in terms of providing support to parents by daughters, which contradicts with previous studies on the topic. This is probably due to the small sample size of rural participants in our sample. 


\section{REFERENCES}

[1] Xi, Z.S. (2020). Marriage story of awakening the world. People's Literature Press.

[2] Sun, Y., (2014). The Role of Daughters in the Aged Care in Rural Families: A Gender Investigation of Intergenerational Relations in Rural Families in Weifang, Shandong. Theory Research, 56(12),79-80.

[3] Li, Y.P. (2021). The Rural "Daughter Endowment" and It's Mechanism from the Perspective of Family Politics. Journal of Nanjing Agricultural University (Social Sciences Edition). 21(1):1-10. DOI: 10.19714/j.cnki.1671-7465.2021.000,p3.

[4] Fan, C.C., \& Xia, Y. (2015). Changes of Daughter's Role in the Rural Family Endowment. Journal of Shanxi Agricultural University (Social Science Edition). 14(2):136-140. DOI:10.13842/j.cnki.issn1671-816x.2015.02.006, p5

[5] Zhu, A.X., \& Gao R., (2016). "Are Parents' Old Age Secured by Having Sons or Daughters?": Views of Chinese Seniors'. Collection of Women's Studies. 25(4):36-44.

[6] Tang C., Ma, C.H, \& Shi J.Q. (2009). Ethic and Fairness of Daughter's Supporting to Her Parents' Family: Gender Study on Intergenerational Family Relation in Rural Areas of Eastern Zhejiang Province. Sociological Studies. 24(6):18-36. DOI:10.19934/j.cnki.shxyj.2009.06.002

[7] Wang, J. (2021). The influence of the number of children and education level on the supply of family endowment resources

[8] Pan, L.Q., \& Zhang, S.H. (2021). The impact of city size on gender wage gap 